\title{
Clinical conundrums in the management of diabetic ketoacidosis in the elderly
}

\author{
Vishal Sehgal, Brian Ulmer \\ St. Vincent Hospital Indianapolis, IN, USA
}

\section{ABSTRACT}

One of the dreaded life-threatening complications of diabetes mellitus (DM) is diabetic ketoacidosis (DKA). American Diabetic Association (ADA) came out with 2018 guidelines on the management of DM and its complications, but these are woefully silent on the clinical conundrums that accompany DKA in elderly patients. In elderly patients, DKA is often complicated by sepsis, atrial fibrillation, polypharmacy, nonketotic hyperosmolar states, atypical clinical presentations, acute kidney injury (AKI), dementia, and noncompliance with medications. Here in we highlight these conundrums that need to be addressed to improve morbidity and mortality in elderly patients.

Key words: elderly patients, diabetic ketoacidosis, diabetes mellitus, healthcare cost, polypharmacy.

\section{INTRODUCTION}

Diabetes mellitus (DM) and its complications cost billions of dollars to the taxpayer. According to the American Diabetes Association (ADA), the cost of DM to the tax payer was $\$ 245$ billion in 2012 . This is a change from $\$ 174$ billion in 2007 when the cost was last examined. Majority of the cost is incurred in the inpatient care of diabetes. The problem gets compounded by the increasingly elderly population that is diabetic. ${ }^{[1]}$ In the United States, more than $25 \%$ of elderly patients are diabetic and the number is rapidly increasing. Shrestha et al. calculated the cost of diabetic ketoacidosis (DKA) related hospitalization to be $\$ 14,236 .{ }^{[2]}$ The cost is higher in elderly patients who have multiple comorbidities and are almost always on polypharmacy. ${ }^{[3,4]}$

National diabetes surveillance program by the Center of Disease Control (CDC) showed that in 2010, DM figured second in the list of top discharge diagnosis of patients aged more than 18 years. Also survey from 1988 to 2009 showed that the discharges with DM as first-listed diagnosis increased from 454,000 to 688,000 . The average length of stay (LOS) for hospital discharges in which DM had been listed as diagnosis decreased to 4.8 days in 2009 from 9.0 days in 1988. This is likely a manifestation of improved inpatient care of DM and related complications. When the data were stratified using age as the criteria, it showed that discharge rates were $19.83 \%$ for patients younger than 45 years, $20.28 \%$ for those aged $45-64$ years, $30.96 \%$ for those aged $65-74$ years, and $47.97 \%$ for those aged 75 years and older. This likely reflects the increased prevalence of DM in the elderly population and also the presence of increased comorbidities that leads to frequent hospitalizations. ${ }^{[5]}$

\section{CLINICAL CONUNDRUMS ASSOCIATED WITH DKA}

The management of DKA has evolved over the years so that inpatient morbidity and mortality has continued to decrease especially after the evolution of low-dose insulin regimen currently being used. ${ }^{[-14]}$ But most data on DKA are based on the younger population with mostly DM1. Elderly patients present with unique problems that add to the complexity with multiple clinical conundrums that accompany DKA (Figure 1). In a retrospective study by 


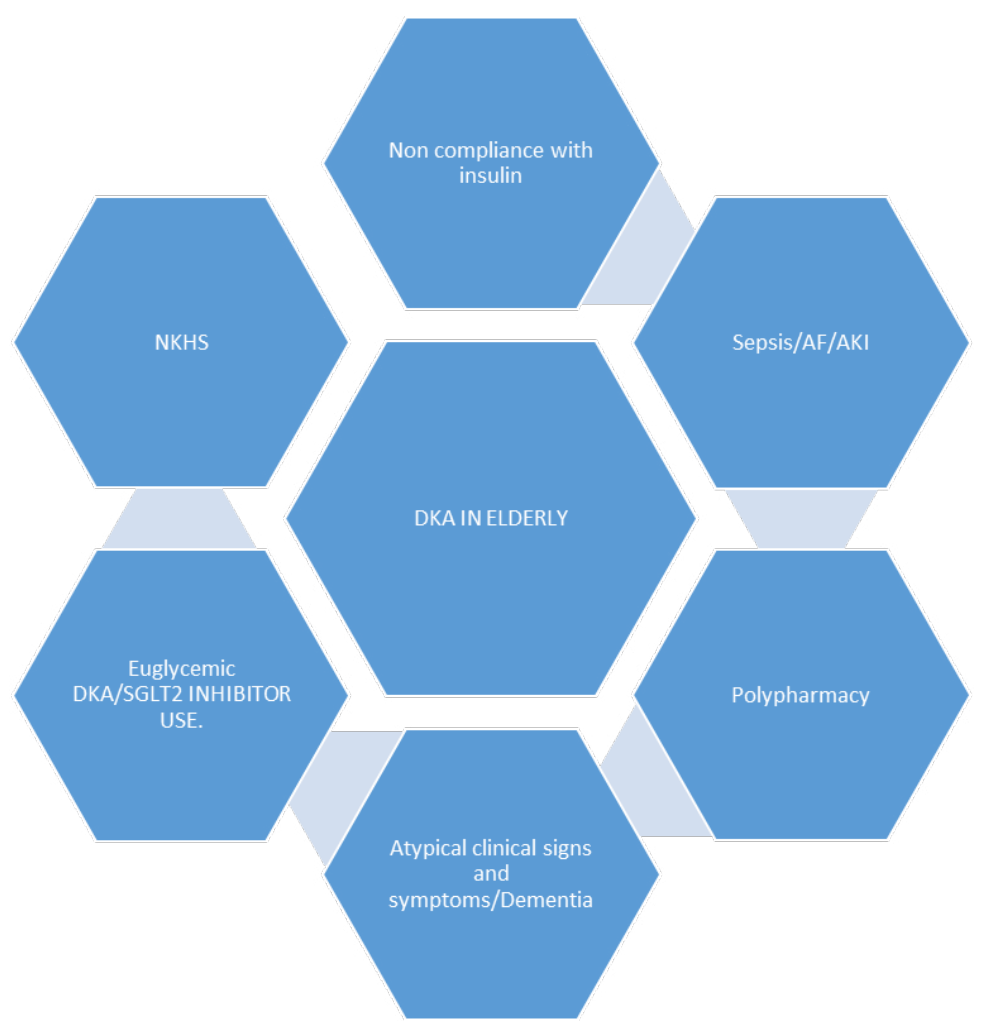

Figure 1: Clinical conundrums in the management of DKA in elderly patients. DKA: diabetic ketoacidosis; AF: atrial fibrillation; AKI: acute kidney injury.

Gilani et al., majority of the admissions were related to the comorbidities and often not because of diabetes. ${ }^{[15]}$ This is often the most complicated part because comorbidities also define the morbidity and mortality associated with DKA. ${ }^{[15]}$

The most common precipitating factors for DKA in elderly patients are related to insulin noncompliance and comorbidities. ${ }^{[16]} \mathrm{It}$ is an arduous task to ensure compliance. Change of insulins has not helped. Lantus and Levemir showed that long-acting insulin has not shown to lower the incidence of DKA as compared to NPH.$^{[17]}$ Insulin Degludec is an ultra-long-acting insulin in the market since 2011. At the outset, it sounds promising as it is longer acting with half-life of $25 \mathrm{~h}$ and sustained action for well beyond $42 \mathrm{~h}$. It has hexadecandioyl fatty acid attached to lysine at the end of the B chain of insulin by glutaryl bond. Also, the last amino acid of the B chain is deleted. This structural modification confers it the property of forming multihexamers when it is injected subcutaneously. Also, it is stabilized in the solution as bihexamers by the addition of phenol and zinc. As zinc and phenol gradually dissipate, there is a gradual and steady release of insulin monomers into the circulation. This gives a sustained and steady action of insulin for roughly beyond $42 \mathrm{~h}$. Its role in the prevention of DKA is yet unexplored but does sound promising given its pharmacodynamic profile.
Comorbidities often define the morbidity and mortality associated with DKA and is the hallmark of DKA in elderly patients. Elderly patients with DM often present with sepsis. DM is an immunosuppressed state and patients with DM remain at increased risk for DM-related infections and sepsis. Sepsis leads to increased reversible secretion of cortisol. ${ }^{[18,19]}$ This leads to stress hyperglycemia, which in patients with impaired beta cell function could potentially precipitate DKA. On the other end of spectrum are patients who are predisposed to hypoglycemia secondary to sepsis. ${ }^{[20-25]}$

Another frequent accompaniment of DKA in elderly patients is atrial fibrillation (AF). It is also observed with increased frequency with aging and more commonly in elderly patients with DM and vice versa. ${ }^{[2]}$ Poor glycemic control in patients with $\mathrm{AF}$ is a sign of poor prognosis in stroke patients. ${ }^{[2]}$ The combo of $\mathrm{AF}$ and $\mathrm{DM}$ acts synergistically in increasing morbidity and mortality associated with $\mathrm{AF} \cdot{ }^{[28,29]}$ Acute $\mathrm{AF}$ is also a stressful state and leads to increased release of stress hormones such as catecholamines, cortisol, and glucagon could potentially cause relative deficiency of insulin.

Polypharmacy is an often-overlooked conundrum that often complicates things in more ways than one. It has 
implicated as one of the causes of noncompliance with medications. It is also an impediment on focusing on drugs that really matter. Healthcare professionals need to proactively reconsider the use of drugs and stop many drugs of questionable benefit. Many cases of DKA could be prevented by proactive communication with healthcare professional and compliance with medications. ${ }^{[1,24,30-32]}$ Polypharmacy also contributes to misdiagnosis of DKA in elderly patients. Nitroprussidebased urine dipsticks are used for quick diagnosis of DKA. Captopril and penicillamine could contribute to false-positive results for DKA. False-negative results are also possible with nitroprusside test as it does not detect $\beta$-hydroxyl butyrate, which is the principal ketone in DKA. The ratio of $\beta$-hydroxybutyrate to acetoacetic acid is $1: 10$ in DKA. The third ketone is acetone, which is technically not an acid and not detected by nitroprusside. This diagnostic dilemma could give false reassurance to the patient and lead to lapse in the window of opportunity when DKA could be potentially detected. Direct assay of $\beta$-hydroxybutyrate levels is more sensitive and specific. ${ }^{[33]}$ But these tests are time consuming and not available in all centers.

Another frequent accompaniment of DKA is nonketotic hyperosmolar state (NKHS). The dose of insulin required to suppress lipolysis and ketosis is about $1 / 10$ th compared to dose required for glucose utilization. Therefore, NKHS often coexists with concomitant DKA.

NKHS, nonketotic hyperosmolar state; AF, atrial fibrillation; AKI, acute kidney injury; SGLT, sodium glucose transporter.

Sodium glucose cotransporter 2 (SGLT2) inhibitor use in high-risk elderly population has been shown to reduce allcause mortality in high-risk population (EMPA-REG). ${ }^{[34,35]}$ Whether this is a class effect or restricted to empagliflozin is not clear. ${ }^{[34]}$ But they need to be used cautiously as they are all implicated in euglycemic ketoacidosis. This could potentially increase the likelihood of DKA by 7-fold and lead to missing the diagnosis of DKA in elderly patients. [36-39] Clinicians need to have low threshold in testing DKA in this group of population because they may be relatively symptomatic in early DKA. ${ }^{[40]}$ The clinical conundrum faced by clinicians is often the diagnosis and management of euglycemic DKA. Low-dose insulin protocol typically recommends starting insulin at a dose of 0.1 units $/ \mathrm{kg}$ after giving a bolus of the same amount. ${ }^{[41]}$ What insulin dose or fluids are needed for resuscitation in SGLT2 inhibitorrelated DKA remains undefined. We recommend starting dose of insulin to be 0.05 units/h and starting dextrosecontaining fluids for rehydration. Again, the timing of last dose of SGLT2 inhibitor would affect the clinical course of the DKA and management.
Nausea, vomiting, and acute abdomen pain are the most common clinical features of DKA. The acuteness of symptoms correlates with the severity of acidosis. ${ }^{[42]}$ But nausea and vomiting are common presentation of multiple disease pathologies, and the diagnosis could be easily missed. In our experience, elderly patients may often have atypical presentation, neurologic symptoms often predominate, and GI symptoms may be surprisingly incipient. Also hyperventilation is frequently observed in severe DKA, which could potentially mislead the clinician towards pulmonary or cardiac dysfunction. ${ }^{[43]}$

Acute kidney injury (AKI) is also a frequent accompaniment of DKA. Glucosuria caused by osmotic diuresis aggravates dehydration and ultimate decline in renal function. This leads to impaired metabolism of many drugs including insulin and all medication use needs to be reassessed.

Elderly patients frequently have underlying dementia and are often on antipsychotics. Patients on antipsychotics have higher incidence of DKA-associated admissions to the hospital. ${ }^{[44-46]}$ Antipsychotics per se have the potential to cause metabolic syndrome. This is more so with atypical antipsychotics that are used more often. When atypical antipsychotics are used in people with DM1, they are not only at higher risk of uncontrolled DM but there is increased incidence of DKA. ${ }^{[44-48]}$ Frequent reassessment are needed to prevent hyperglycemic complications related to atypical antipsychotics.

\section{CONCLUSION}

DKA in elderly patients is often masked by various clinical conundrums, which may mask the disease pathology and lead to a delay in the diagnosis and management of DKA. Clinical guidelines aimed specifically addressing the management of DKA in elderly patients could go a long way in reducing the morbidity, mortality, and cost associated with management of DKA and its associated clinical conundrums.

\section{Conflict of Interest}

None declared.

\section{REFERENCES}

1. Sehgal V, Bajwa SJ, Sehgal R, Eagan J, Reddy P, Lesko SM. Predictors of acute kidney injury in geriatric patients undergoing total knee replacement surgery. Int J Endocrinol Metab 2014; 12: e16713.

2. Shrestha SS, Zhang P, Barker L, Imperatore G. Medical expenditures associated with diabetes acute complications in privately insured U.S. youth. Diabetes Care 2010; 33: 2617-22.

3. Sehgal V, Bajwa SJ, Sehgal R, Bajaj A, Khaira U, Kresse V. Polypharmacy and potentially inappropriate medication use as the precipitating factor 
in readmissions to the hospital. J Family Med Prim Care 2013; 2: 194-9.

4. Freire AX, Umpierrez GE, Afessa B, Latif KA, Bridges L, Kitabchi AE. Predictors of intensive care unit and hospital length of stay in diabetic ketoacidosis. J Crit Care 2002; 17: 207-11.

5. Rodrigo E, Santos L, Piñera C, Eagan J, Reddy P, Lesko SM. Prediction at first year of incident new-onset diabetes after kidney transplantation by risk prediction models. Diabetes Care 2012; 35: 471-3.

6. Yu CH, Straus S, PhD RB. The ABCs of DKA: Development and Validation of a Computer-Based Simulator and Scoring System. J Gen Intern Med 2015; 30: 1319-32.

7. Gosmanov AR, Gosmanova EO, Dillard-Cannon E. Management of adult diabetic ketoacidosis. Diabetes Metab Syndr Obes 2014; 7: 255-64.

8. Basnet S, Venepalli PK, Andoh J, Verhulst S, Koirala J. Effect of normal saline and half normal saline on serum electrolytes during recovery phase of diabetic ketoacidosis. J Intensive Care Med 2014; 29: 38-42.

9. Jovanovic A, Stolic RV, Rasic DV, Markovic-Jovanovic SR, Peric VM. Stroke and diabetic ketoacidosis--some diagnostic and therapeutic considerations. Vasc Health Risk Manag 2014; 10: 201-4.

10. Suwarto S, Sutrisna B, Waspadji S, Pohan HT. Predictors of five days mortality in diabetic ketoacidosis patients: a prospective cohort study. Acta Med Indones 2014; 46: 18-23.

11. Umpierrez GE, Kitabchi AE. Diabetic ketoacidosis: risk factors and management strategies. Treat Endocrinol 2003; 2: 95-108.

12. Kitabchi AE, Nyenwe EA. Hyperglycemic crises in diabetes mellitus: diabetic ketoacidosis and hyperglycemic hyperosmolar state. Endocrinol Metab Clin North Am 2006; 35: 725-51, viii.

13. Nyenwe EA, Wan JY, Kitabchi AE. Venous serum bicarbonate concentration predicts arterial $\mathrm{PH}$ in adults with diabetic ketoacidosis. Endocr Pract 2014; 20: 201-6.

14. Edo AE. Clinical profile and outcomes of adult patients with hyperglycemic emergencies managed at a tertiary care hospital in Nigeria. Niger Med J 2012; 53: 121-5.

15. Gillani SM, Aziz U, Blundell D, Singh BM. Non elective re-admissions to an acute hospital in people with diabetes: Causes and the potential for avoidance. The WICKED project. Prim Care Diabetes 2015; 9: 392-6.

16. Barski L, Nevzorov R, Rabaev E, Jotkowitz A, Harman-Boehm I, Zektser $\mathrm{M}$, et al. Diabetic ketoacidosis: clinical characteristics, precipitating factors and outcomes of care. Isr Med Assoc J 2012; 14: 299-303.

17. Karges B, Kapellen T, Neu A, Hofer SE, Rohrer T, Rosenbauer J, et al. Long-acting insulin analogs and the risk of diabetic ketoacidosis in children and adolescents with type 1 diabetes: a prospective study of 10,682 patients from 271 institutions. Diabetes Care 2010; 33: 1031-3.

18. Pichardo-Lowden A, Gabbay RA. Management of hyperglycemia during the perioperative period. Curr Diab Rep 2012; 12: 108-18.

19. Dorin RI, Qualls CR, Torpy DJ, Schrader RM, Urban FK. Reversible increase in maximal cortisol secretion rate in septic shock. Crit Care Med 2015; 43: 549-56.

20. Storey S, Von Ah D. Impact of malglycemia on clinical outcomes in hospitalized patients with cancer: a review of the literature. Oncol Nurs Forum 2012; 39: 458-65.

21. Mendoza A, Kim YN, Chernoff A. Hypoglycemia in hospitalized adult patients without diabetes. Endocr Pract 2005; 11: 91-6.

22. Kagansky N, Levy S, Rimon E, Cojocaru L, Fridman A, Ozer Z, et al. Hypoglycemia as a predictor of mortality in hospitalized elderly patients. Arch Intern Med 2003; 163: 1825-9.

23. Shilo S, Berezovsky S, Friedlander Y, Sonnenblick M. Hypoglycemia in hospitalized nondiabetic older patients. J Am Geriatr Soc 1998; 46: 978-82.

24. Fasanmade OA, Odeniyi IA, Ogbera AO. Diabetic ketoacidosis: diagnosis and management. Afr J Med Med Sci 2008; 37: 99-105.

25. Brodovicz KG, Mehta V, Zhang Q, Zhao C, Davies MJ, Chen J, et al. Association between hypoglycemia and inpatient mortality and length of hospital stay in hospitalized, insulin-treated patients. Curr Med Res Opin 2013; 29: 101-7.
26. Proietti R, Russo V, Wu MA, Maggioni AP, Marfella R. [Diabetes mellitus and atrial fibrillation: evidence of a pathophysiological, clinical and epidemiological association beyond the thromboembolic risk]. G Ital Cardiol (Rome) 2017; 18: 199-207.

27. Kim JS, Kim RY, Cha JK, Rha HW, Kang MJ, Kim DH, et al. Pre-stroke glycemic control is associated with early neurologic deterioration in acute atrial fibrillation-related ischemic stroke. eNeurologicalSci 2017; 8: $17-21$.

28. Fumagalli S, Said SA, Laroche C, Gabbai D, Boni S, Marchionni N, et al. Management and prognosis of atrial fibrillation in diabetic patients: an EORP-AF General Pilot Registry report. Eur Heart J Cardiovasc Pharmacother 2017; 4:172-9.

29. Akirov A, Grossman A, Shochat T, Shimon I. Hyperglycemia on admission and hospitalization outcomes in patients with atrial fibrillation. Clin Cardiol 2017; 40: 1123-8.

30. Morris LR, Kitabchi AE. Efficacy of low-dose insulin therapy for severely obtunded patients in diabetic ketoacidosis. Diabetes Care 1980; 3: 53-6.

31. Jefferies CA, Nakhla M, Derraik JG, Gunn AJ, Daneman D, Cutfield WS. Preventing Diabetic Ketoacidosis. Pediatr Clin North Am 2015; 62: 857-71.

32. Sehgal V, Bajaj A, Sehgal R, Bajwa SJ, Khaira U, Kresse V. Polypharmacy and potentially inappropriate medication use as the precipitating factor in readmissions to the hospital. J Family Med Prim Care 2013; 2:194-9.

33. Yang HL, Yuan GP, Deng H, Chen C, Peng X, An ZM. [The diagnostic value of serum beta-hydroxybutyrate in diabetic ketosis or diabetic ketoacidosis]. Sichuan Da Xue Xue Bao Yi Xue Ban. 2014; 45: 270-3.

34. Tang H, Fang Z, Wang T, Cui W, Zhai S, Song Y. Meta-Analysis of Effects of Sodium-Glucose Cotransporter 2 Inhibitors on Cardiovascular Outcomes and All-Cause Mortality Among Patients With Type 2 Diabetes Mellitus. Am J Cardiol 2016; 118: 1774-80.

35. Zinman B, Wanner C, Lachin JM, Fitchett D, Bluhmki E, Hantel S, et al. Empagliflozin, Cardiovascular Outcomes, and Mortality in Type 2 Diabetes. N Engl J Med 2015; 373: 2117-28.

36. Bhatnagar RK, Kurera I, Perry R, Tringham J. Euglycaemic DKA secondary to Canaglifozin, an easily missed diagnosis. Acute Med 2017; 16: 196-9.

37. Brown F, McColl T. Euglycemic Diabetic Ketoacidosis Secondary to Dapagliflozin Use: A Case Report. J Emerg Med 2018; 54: 109-11.

38. Kelmenson DA, Burr K, Azhar Y, Reynolds P, Baker CA, Rasouli N. Euglycemic Diabetic Ketoacidosis With Prolonged Glucosuria Associated With the Sodium-Glucose Cotransporter-2 Canagliflozin. J Investig Med High Impact Case Rep 2017; 5: 2324709617712736.

39. Blau JE, Tella SH, Taylor SI, Rother KI. Ketoacidosis associated with SGLT2 inhibitor treatment: Analysis of FAERS data. Diabetes Metab Res Rev. 2017; 33.[Epub 2017 Sep 29]

40. Andrews TJ, Cox RD, Parker C, Kolb J. Euglycemic Diabetic Ketoacidosis with Elevated Acetone in a Patient Taking a Sodium-Glucose Cotransporter-2 (SGLT2) Inhibitor. J Emerg Med 2017; 52: 223-6.

41. Kitabchi AE, Murphy MB, Spencer J, Matteri R, Karas J. Is a priming dose of insulin necessary in a low-dose insulin protocol for the treatment of diabetic ketoacidosis? Diabetes Care 2008; 31: 2081-5.

42. Umpierrez G, Freire AX. Abdominal pain in patients with hyperglycemic crises. J Crit Care 2002; 17: 63-7.

43. Cefalu WT. Diabetic ketoacidosis. Crit Care Clin 1991; 7: 89-108.

44. Galler A, Bollow E, Meusers M, Bartus B, Näke A, Haberland H, et al. Comparison of glycemic and metabolic control in youth with type 1 diabetes with and without antipsychotic medication: analysis from the nationwide German/Austrian Diabetes Survey (DPV). Diabetes Care 2015; 38: 1051-7.

45. Ely SF, Neitzel AR, Gill JR. Fatal diabetic ketoacidosis and antipsychotic medication. J Forensic Sci 2013; 58: 398-403.

46. Guenette MD, Hahn M, Cohn TA, Teo C, Remington GJ. Atypical antipsychotics and diabetic ketoacidosis: a review. Psychopharmacology (Berl) 2013; 226: 1-12. 
Sehgal and Ulmer: DKA in the elderly

47. Hara JS, Rahbar AJ, Jeffres MN, Izuora KE. Impact of a hyperglycemic crises protocol. Endocr Pract 2013; 19: 953-62.

48. Heal DJ, Gosden J, Jackson HC, Cheetham SC, Smith SL. Metabolic consequences of antipsychotic therapy: preclinical and clinical perspectives on diabetes, diabetic ketoacidosis, and obesity. Handb Exp Pharmacol 2012; (212): 135-64.
How to cite this article: Sehgal V, Ulmer B. Clinical conundrums in the management of diabetic ketoacidosis in the elderly. J Transl Int Med 2019; 7: 10-4. 